\title{
COMPONENTE TECNOLÓGICO: ARTICULACIÓN CON EL ÁREA EDUCACIÓN FÍSICA PARA PROMOVER LA FORMACIÓN INTEGRAL EN LA ESCUELA
} \section{TECHNOLOGICAL COMPONENT: ARTICULATION WITH PHYSICAL EDUCATION AREA TO PROMOTE INTEGRAL TRAINING AT SCHOOL}

\section{Nicol Pinilla Fonseca ${ }^{1}$}

Miembro Grupo de Investigación Ambientes de Enseñanza-Aprendizaje de las Ciencias Básicas y Sociales (AMECl)

\section{María Cristina Gamboa Mora ${ }^{2}$}

\section{Gerardo Patiño Varón ${ }^{3}$}

Universidad de Baja California. Tepic-México

\section{RESUMEN}

El proyecto tuvo como objetivo establecer 1 Institución Educativa San Isidoro. Espinal (Tolima), Colombia. Doctoranda Universidad de Baja California. Tepic-México. https://orcid.org/0000-0003-1375-3389 nicolpinillafonseca@gmail.com

Teléfono +57 3114505385 Ibagué - Tolima (Colombia) 2 Universidad Nacional abierta y a Distancia, Colombia. Doctora en Innovación e investigación en didáctica https://orcid.org/0000-0001-8459-099X

maria.gamboa@unad.edu.co o mcgamboam@udistrital. edu.co Teléfono +57 3168702204 Bogotá (Colombia) 3 Doctorando Universidad de Baja California. TepicMéxico. https://orcid.org/0000-0001-5450-2864 gerardopatinovaron@gmail.com Teléfono +57 3107897352 Ibagué - Tolima (Colombia) la percepción sobre la formación integral de los estudiantes de una institución educativa de Tolima-Colombia, obtenida tras la implementación de estrategias inclusivas de tecnología y Tecnologías de la Información y la Comunicación, con procesos motrices y corporales. El diseño de investigación fue cuasiexperimental, participaron 200 estudiantes de media, se propuso un instrumento tipo escala Likert pretest-postest y se comprobó la efectividad, a través de la prueba $t$ de student. Se concluye que las estrategias empleadas 
tienen efecto sobre la formación integral, ya que las medias de percepción aumentaron significativamente.

\section{PALABRAS CLAVE:}

Formación Integral, Educación Física, Tecnología y TIC.

\section{ABSTRACT}

The aim of this project was to establish a perception of the comprehensive training that some students had from a school in TolimaColombia and what their perception was after the implementation of inclusive strategies of technology and Information and Communication Technologies, applying motor and physical processes. The research design was quasiexperimental. An average of 200 students from middle school participated. Moreover, a pre and post test Likert scale instrument was proposed, and its effectiveness was tested by a student's $\mathrm{t}$-test. It was concluded that the strategies used had an effect on comprehensive training, since the perception means increased significantly.

\section{KEY WORD:}

Comprehensive Training, Physical Education, Technology and ICT.

\section{INTRODUCCIÓN}

El siglo XXI se ha distinguido por el dinámico y colosal avance de las Nuevas Tecnologías, dando lugar a lo que hoy se conoce como la sociedad de la información (Fernández y Ladrón, 2015). En razón a ello, el papel que desempeña la Tecnología es el de impulso al cambio social y no solo en el aspecto de información, sino además como agente posibilitador de la comunicación humana. Al respecto, Buckingham (2002; citado por Barquero y Calderón, 2016), sostiene que el proceso evolutivo de la tecnología engrana procesos tanto de información como de comunicación humana, mostrando los efectos positivos y negativos de la misma. Es imposible hoy tratar temas educativos o esperar generar procesos de enseñanza- aprendizaje exitosos y/o significativos, basados en modelos o esquemas educativos tradicionales los cuales están separados completamente del uso de la Tecnología y las Tecnologías de la Información y las comunicaciones en adelante TIC en el aula, ya que es evidente que los jóvenes tienen un innato contacto con estas herramientas y en muchos casos se reemplazan actividades por el uso de dispositivos móviles, redes sociales, videojuegos, internet entre muchos otros instrumentos disponibles y de fácil acceso para ellos. Es aquí donde se evidencia la necesidad de incluir tanto la Tecnología como las TIC en los procesos del aula, pues ellas hacen más dinámicas las clases y permiten que se genere mayor nivel de aprehensión no sólo de conocimientos sino de habilidades que permiten que el estudiante se desarrolle de manera integral.

La Tecnología hace parte fundamental del proceso de globalización y su consolidación en el mundo establece cambios sociales, políticos, culturales y económicos para las distintas comunidades, generando influencia directa en el desarrollo de los seres humanos y en la forma como ven y perciben el mundo a partir del uso de la misma. La evolución de la sociedad y de los sistemas de comunicación han redireccionado el sentir de la educación hacia un camino en el que las TIC se encuentran a la vanguardia y posibilitan al ser humano tener el mundo en sus manos. En razón a lo anterior, las TIC permiten que hoy en día la educación llegue a más personas, más lugares y obviamente con una mayor facilidad, generando de esta manera un beneficio en el que no solo ganan los estudiantes, los docentes, sino que finalmente gana la sociedad. En este sentido, Fernández y Ladrón (2015) sostienen que los avances científicos y tecnológicos están provocando que la sociedad esté cambiando a un ritmo vertiginoso. $Y$ es en 
este cambio en el que los docentes debemos ser parte y facilitadores de estrategias que permitan competir en la sociedad a través del uso de la Tecnología.

Si bien es cierto que el área de educación física influencia el aspecto motor del ser humano, involucrando movimientos y habilidades físicas que propenden por su desarrollo tanto físico como social, no debe aislarse de la evolución social ni a los avances tecnológicos, mucho menos a las nuevas exigencias educativas y los nuevos retos que se promueven en la sociedad actual con la incursión de la Tecnología y las TIC en el aula, por el contrario el reto principal consiste en establecer mecanismos o estrategias que permitan que estas hagan parte del área y contribuyan con el mejoramiento del ser humano tanto en su aspecto físico como en su actuar en la sociedad, en este sentido, Fernández, Herrera y Navarro (2015) manifiestan que la realización de actividades con apoyo de las TIC, constituye un importante aporte de esta disciplina a la consolidación de los procesos educativos de los estudiantes. El problema radica, en cómo incluir en la educación física actividades que impliquen el uso del componente Tecnológico para formar integralmente a los jóvenes y que adicionalmente se generen aprendizajes significativos, para resolver esta situación, se realiza una investigación, en la que se aplican estrategias específicas como la creación de un blog, el uso de aplicaciones móviles para desarrollar la clase, prácticas educativas en las que se tenga la opción de usar nuevas tecnologías, uso de redes sociales que permitan la difusión de información relevante del área, grabación de vídeos educativos a través de sus dispositivos móviles entre otras, hacen parte de las estrategias empleadas para lograr una inclusión del componente en el área y de esta manera lograr el objetivo de establecer la percepción sobre el grado de formación integral que vienen logrando en el desarrollo de un curso de educación física con la incorporación del componente tecnológico, los estudiantes de media de la institución educativa San Isidoro del municipio de Espinal (Tolima - Colombia).

\section{REFERENTES TEÓRICOS}

\section{TECNOLOGÍA EN EDUCACIÓN FÍSICA}

La Tecnología es un saber que se desarrolla a través de una relación teórico-práctica que permite logros de calidad en los procesos aplicados a objetos e instrumentos (aquello que sirve para algo) tecnológicos y a la producción de bienes o servicios con el fin de solucionar problemas y necesidades humanas (Soto, 2000). Para el caso de un área como la educación física, la tecnología se ve reflejada en la calidad de materiales como balones, colchonetas, instrumentos de medición específica para cada disciplina deportiva, o en instrumentos de clase simples como pitos o cronómetros que permiten ser más dinámica la clase. Al respecto, Rojano (2010) sostiene que actualmente existen nuevos y modernos recursos tecnológicos que son utilizados para realizar mediciones en el ámbito deportivo. Todo ello producto de la notable evolución de la sociedad y sobre todo del conocimiento, pues es innegable que sólo a través de ellos el ser humano se plantea nuevos desafíos y es capaz de dar respuesta de forma más inmediata a sus necesidades. En este sentido, Hernández (2017) afirma que uno de los factores que se considera relacionado entre conocimiento y sociedad es la tecnología, proceso que se ve reflejado en distintas ciencias, ocasionando oportunidades de cambio y adaptación, pero al mismo tiempo desafíos para la humanidad. Entre tanto, Rojano (2010) manifiesta que la incorporación de la tecnología en la sociedad ha sucedido a un ritmo tan acelerado, que su influencia es muy importante para la sociedad, por lo que se hace absolutamente necesario tener un conocimiento al menos básico de la misma para lograr que exista un desarrollo completo del entorno. Evidentemente la tecnología desarrolla 
un papel significativamente importante dentro del desarrollo de la sociedad, pues es a través de ella que se promueve una relación entre el conocimiento, la ciencia y las necesidades humanas en el sentido de adaptación y mejora.

La Tecnología se ha convertido en un aspecto central de coyunturas, que brindan la posibilidad de generar innovación en la educación, donde los resultados obtenidos, de esta intervención deberán buscar la solución a problemas tanto sociales como educativos útiles para el desarrollo humano (Hernández, 2017). Es decir, que la Tecnología debe responder a las demandas sociales y culturales de la actualidad $y$ es en este punto, donde la educación debe generar espacios de inclusión de este componente en beneficio del ser humano. En razón a lo anterior, Plaza (2013) plantea que la Tecnología aplicada a la educación genera impactos tanto en los aspectos de carácter pedagógico, administrativo y de gestión, como en los tipos de competencias reclamadas por la sociedad. Por ello, el sistema educativo se ve en la imperante obligación de integrar las nuevas tecnologías como consecuencia de la actual necesidad social reflejada en la escuela, que debe adaptarse y evolucionar al mismo ritmo y con ello el área de Educación Física como una asignatura más dentro del currículo básico oficial (Fernández y Ladrón, 2015). Entonces, es necesario que la escuela evolucione a la par con la Tecnología y se valga de ella para mejorar los procesos de enseñanza -aprendizaje de los estudiantes, que como nativos digitales utilizan las herramientas tecnológicas en el proceso de aprendizaje (Hernández, 2017) y en este orden de ideas la escuela debería permitir y adecuar los espacios para que este proceso sea más efectivo en el aula y fuera de ella.

La inclusión de la Tecnología en la clase de educación física, no es un proceso que debe hacer solo el estudiante, pues es el maestro el llamado a guiarlo y orientarlo en el uso adecuado de la misma, generando una formación de tipo integral en el aula. Por otra parte, el problema no es la tecnología sino el uso que se hace de la misma (Quintero, 2018), los estudiantes en educación media están en proceso de toma de decisiones o de maduración y suelen hacer uso de la misma o se involucran con fines recreativos, más que con una conciencia del aporte de esta al proceso académico. De acuerdo a lo anterior, Camblor (2014) infiere que en el ámbito educativo se puede usar la tecnología en la enseñanza de la Educación Física, con el objetivo de lograr un acercamiento empático con el estudiante, acompañando su proceso de transformación y haciendo uso de un elemento inherente a su contexto actual. Este acercamiento desencadena en un estudiante formado integralmente, con una competencia tecnológica estructurada de manera que va a ritmo con la evolución de la sociedad.

\section{TIC Y EDUCACIÓN FÍSICA}

Las TIC, son herramientas que permiten crear de una manera más rápida llegando a más usuarios y lugares lejanos. En este sentido, Prat, Camerino y Coiduras (2013) sostienen que el uso de las herramientas tecnológicas en el aula representa en el docente una amplia gama de ventajas en relación a los procesos de enseñanza y aprendizaje debido a la flexibilidad, inmediatez, adaptabilidad, interactividad y la combinación de múltiples experiencias que contribuyen a mejorar este proceso. Con la revolución tecnológica actual, se generan nuevos recursos que posibilitan y acercan al estudiante a generar un aprendizaje más significativo, por ello la educación debe evolucionar al ritmo de la sociedad.

La inclusión de las TIC al área de Educación Física constituye un desafío para los docentes ya que se vivencia una reforma pedagógica que conlleva a mejorar los procesos de conexión a la actividad física y el deporte (Monroy, 2010; 
citado por Fernández y Ladrón, 2015). Esta mejora en los procesos se da en la medida que el estudiante accede más rápido y fácil tanto a la información como a los medios para conseguirla. Al respecto, Camblor (2014) establece que con la ampliación del uso de las TIC se constituyen nuevos lenguajes, con los que los estudiantes generan empatías tanto cognitivas como expresivas y nuevos modos de percibir el mundo que les rodea. Pero dicha inclusión de las TIC debe iniciar paulatinamente, partiendo del diálogo y compromiso de los estudiantes para utilizarlas en el aula con carácter didáctico (Quintero, 2018). No se debe desconocer que los estudiantes actualmente pasan más tiempo en actividades relacionadas con el uso de las TIC que realizando algún tipo de actividad física, por ello el área debe valerse de las mismas para involucrar a los jóvenes en sus procesos, planteando clases más dinámicas y atractivas. En este sentido, Camblor (2014) manifiesta que emplear herramientas o plataformas informáticas en el aula contribuye con el desarrollo del trabajo en grupo y de proyectos comunitarios en el que se obtienen recursos y actividades que se pueden implementar en la clase de Educación Física, con el fin de promover espacios de mejoramiento en los procesos de enseñanzaaprendizaje del área.

Actualmente, los móviles, los ordenadores portátiles, las tabletas y demás aparatos tecnológicos constituyen una herramienta fundamental en la vida cotidiana de los seres humanos (Fernández y Ladrón, 2015). Por ello, no deben ser extrañas al área de educación física y se deben implementar actividades que incluyan su uso de manera responsable y bajo la supervisión del docente. Al respecto, Quintero (2018) sostiene que el principal aporte de los dispositivos móviles es que se pueden utilizar en cualquier momento y espacio, lo cual abre nuevas posibilidades didácticas a los docentes de Educación Física para hacer uso de las TIC combinadas con la actividad física y deportiva. Así mismo, Aznar, Cáceres, Trujillo y Romero (2019) plantean que los dispositivos móviles son un poderoso recurso para mejorar y aumentar la práctica deportiva, ya que permiten generar y establecer nuevos componentes motivacionales para realizar deporte y actividad física, incidiendo positivamente en el proceso de enseñanza-aprendizaje de la educación física. Existen aplicaciones móviles que contribuyen con la inclusión de las TIC en el aula, con las que el estudiante realiza actividad física y son llamativas para ellos, tal es el caso de la App Munzee, la cual emplea códigos QR y el GPS del dispositivo móvil para hacer uso de ella.

Por otro lado, el uso de redes sociales permite que los estudiantes generen procesos sociales de una manera distinta y se debe aprovechar ello, para fomentar habilidades de manera controlada y en condiciones que permitan generar ambientes de aprendizaje didácticos e innovadores.

\section{FORMACIÓN INTEGRAL}

La formación integral en Latinoamérica viene siendo abordada desde finales del siglo XIX, como respuesta a una serie de inquietudes y situaciones de orden tanto global como local manifestadas en muchos aspectos de la vida, que requieren soluciones en las cuales la educación debe tener un papel primordial. En este sentido, Equihua (2007) sostiene que la formación integral parte del desarrollo equilibrado y armónico de todos los aspectos que llevan al ser humano a formarse intelectual, social, cultural y profesionalmente. La formación integral entonces es entendida, como la formación de todos los aspectos que conforman el ser humano, teniendo en cuenta la complejidad que ello implica. Por otra parte, Campo (1999) considera que la formación integral es un componente fundamental de la condición humana, que es propia de cada una y se produce a partir del proceso propio de la vida. Entre tanto, Orozco (2008) manifiesta que 
la formación integral contribuye a enriquecer el proceso de socialización del estudiante, lo convierte en un ser más sensible a través del desarrollo de sus habilidades artística, morales y la apertura del pensamiento crítico. Es posible afirmar que la formación integral está estrechamente ligada con la acción que ejerce cualquier cultura para desarrollar o potenciar todas las dimensiones propias del ser humano visto como un todo, además de ser considerada como una forma de educar en la que se debe hacer énfasis en el proceso de construcción personal, reconociendo todas las particularidades del ser humano presentes en los diferentes ámbitos de su existencia tanto individual como social. Desde esta perspectiva el proceso educativo debe estar centrado en el estudiante, para que, a través de este, haga uso de todas sus potencialidades, constituya y desarrolle su ser personal.

\section{COMPETENCIAS BLANDAS}

Las competencias blandas hacen referencia al desarrollo de habilidades no cognitivas, que se consideran necesarias para desempeñar de manera adecuada una labor, encaminadas al desarrollo de aptitudes sociales. El desarrollo de dichas competencias $\mathrm{o}$ habilidades le permitirá al ser humano transfigurarse en un individuo más completo e integral. Por otra parte, la Organización Mundial de la Salud las define como un conjunto de habilidades profesionales y relacionales, de carácter social y emotivo, consideradas necesarias para guiar las relaciones entre el individuo y el mundo en el que vive (Tapia, 2018). Al ser de carácter social es innegable que la escuela está en la obligación de propender por el desarrollo de las mismas y brindar al estudiante la posibilidad de que mejore su aspecto emocional, interpersonal y a su vez la interacción que realiza con la sociedad. En este sentido, (Banco Interamericano de Desarrollo, BID, 2017; citado por Gómez, 2019) afirma que las habilidades socioemocionales contribuyen a que los seres humanos identifiquen y manejen sus propias emociones y sean un poco más conscientes de las de los demás. Estas habilidades son consideradas como igual 0 más importantes que las habilidades cognitivas para desempeñarse exitosamente en todos los aspectos de la vida (Organización para la Cooperación y el Desarrollo Económico, OCDE, 2015; citado por García, 2018). Al respecto, Singer, Guzmán y Donoso (2009) sostienen que las competencias blandas son mucho más relevantes que las competencias duras. Debido a la naturaleza de las competencias blandas es posible que el estudiante las desarrolle a lo largo de su vida. Entre tanto, Gómez (2019) plantea que las habilidades se desarrollan tanto en un ambiente formal de educación, como en diferentes contextos. Es decir que el estudiante las puede adquirir en cualquier contexto en el que se desarrolle, pero obviamente en la escuela se deben reforzar.

Dentro de los retos que tiene las instituciones educativas ahora, se encuentran el preparar a los estudiantes en competencias blandas necesarias, para el momento en el que se involucre al campo laboral (Gómez, 2019), no sólo para que sean productivos, también en el aspecto social para que desde sus posibilidades puedan construir cada día una sociedad mejor. Al respecto, Singer, Guzmán y Donoso (2009) argumentan que toda competencia dura puede ser entrenada rápidamente, mientras que una blanda puede tomar más tiempo. Pueden ser forjadas en algún momento de la vida por el ser humano ya que, de acuerdo con, García (2018) son habilidades que no son fijas, sino que por el contrario se pueden aprender a desarrollar. Lo que se debe tener en cuenta según, Ortega, Febles y Estrada (2016) es que una persona tendrá más facilidad que otra al momento de desarrollarlas. 


\section{PERCEPCIONES}

La percepción hace referencia a la función psíquica, que permite a través de los sentidos recibir, elaborar e interpretar la información que proviene del entorno, generando el primer proceso cognoscitivo mediante el cual los seres humanos captan la información (Bustos, 2014). Entre tanto, Rivera (1995) sostiene que la percepción es la fuente de información primaria para el organismo y que aquella información está directamente relacionada con el medio y la situación dentro de ese. Las percepciones básicamente vienen siendo el resultado de una o más experiencias significativas del ser humano en determinado entorno, que hace fijar una idea o característica específica de cada situación. Al respecto, Dunn y Dunn (1985; citado por Gamboa, Briceño y Camacho, 2015) establecen que los seres humanos perciben el mundo a través de tres canales: visual, auditivo y kinestésico. Es decir que las experiencias relevantes van a provenir del contacto a través de lo que el ser humano interpreta de lo que ve, escucha y siente en su corporeidad, generando experiencias subjetivas con la implicación de establecer una evaluación de algo o alguien en determinada situación de aprendizaje (Eiser, 1989; citado por Bustos, 2014). De aquí la importancia de las percepciones, pues es a través de ellas que el ser humano se predispone para generar cualquier tipo de aprendizaje.

De acuerdo, con Mateos (2009) la percepción de una situación puede cambiar de un momento a otro, partiendo de lo que suceda, se haga, el pensamiento que se tenga y los actos de los demás, entre otros aspectos. Ello ocurre en razón a que los seres humanos son distintos y no tiene las mismas necesidades e ideas comunes y cada uno construye significados del mundo a partir de sus propias experiencias y vivencias. Conocer las percepciones u opiniones de los estudiantes sobre el docente y sus actuaciones, ayuda sin lugar a dudas a valorar la correspondencia entre lo que se planifica y lo que finalmente se proyecta (Mateos, 2009).

Con relación a la percepción en el ámbito escolar, se da en el proceso de estar en la escuela, en la interacción con la estructura, mecanismos, contenidos e individuos que hacen parte de esa experiencia (Rivera, 1995). En este sentido, Mateos (2009) afirma que la percepción de los estudiantes sobre la institución escolar influye directamente en los aspectos propios de la dinámica del proceso escolar, tales como su rendimiento académico, integración escolar, relaciones sociales y, en general, en su socialización. El maestro juega un papel determinante en el proceso de percepción de los estudiantes, pues de la imagen que este proyecte sobre los estudiantes depende el resultado de sus procesos de enseñanza ya que, si los estudiantes están cómodos y a gusto con el docente seguramente vincularan esa percepción a la adquisición de nuevos conocimientos y a su proceso de formación.

\section{METODOLOGÍA}

La investigación fue de tipo experimental con diseño cuasiexperimental, desarrollada en la IE San Isidoro institución educativa pública del municipio de Espinal departamento del Tolima (Colombia), la cual atiende población de estrato socioeconómico bajo, con una gran diversidad cultural, los ingresos de la institución provienen del sistema general de participación, los cuales resultan insuficientes para atender en su totalidad las necesidades básicas de infraestructura tecnológica que requiere para los casi 3000 estudiantes de preescolar a grado once en las tres jornadas (mañana, tarde y sabatina). La población correspondió a 200 estudiantes de grados décimo y undécimo (media) de la jornada mañana, los cuales se encuentran en una edad entre los 14 y 17 años, cuentan con una sala de sistemas con 30 equipos de cómputo para desarrollar sus actividades académicas, no cuentan con acceso libre a internet lo cual 
aumenta la brecha tecnológica con relación a los planteles privados y/o de la zona capital del departamento, la principal fuente de ingresos económicos de las familias es agrícola y el turismo en la celebración de las festividades culturales y religiosas propias de la región en el mes de junio.
Se aplicó como pretest un instrumento tipo escala Likert al cual se asignaron las categorías nunca, casi nunca, ocasionalmente, casi siempre y siempre con una escala numérica de 1 a 5 respectivamente, para cada ítem de acuerdo con la descripción de la tabla 1.

Tabla 1. Pretest

\begin{tabular}{|c|c|c|c|c|c|c|}
\hline ÍTEM & ENUNCIADO & NUNCA & $\begin{array}{c}\text { CASI } \\
\text { NUNCA }\end{array}$ & OCASIONALMENTE & $\begin{array}{c}\text { CASI } \\
\text { SIEMPRE }\end{array}$ & SIEMPRE \\
\hline 1 & $\begin{array}{l}\text { Utiliza las TIC en el } \\
\text { desarrollo de sus labores } \\
\text { académicas. }\end{array}$ & 1 & 2 & 3 & 4 & 5 \\
\hline 2 & $\begin{array}{l}\text { Emplea la Tecnología } \\
\text { como complemento de sus } \\
\text { aprendizajes. }\end{array}$ & 1 & 2 & 3 & 4 & 5 \\
\hline 3 & $\begin{array}{l}\text { Participa en foros, chats, } \\
\text { grupos y/o redes sociales } \\
\text { cuyas temáticas aportan a } \\
\text { su desarrollo personal. }\end{array}$ & 1 & 2 & 3 & 4 & 5 \\
\hline 4 & $\begin{array}{l}\text { Se le facilita buscar, } \\
\text { seleccionar, evaluar y } \\
\text { organizar información en } \\
\text { entornos digitales. }\end{array}$ & 1 & 2 & 3 & 4 & 5 \\
\hline 5 & $\begin{array}{l}\text { Transmite información } \\
\text { relevante a través de las } \\
\text { redes sociales. }\end{array}$ & 1 & 2 & 3 & 4 & 5 \\
\hline 6 & $\begin{array}{l}\text { Conoce y respeta los } \\
\text { límites legales, culturales } \\
\text { y éticos de compartir } \\
\text { información por Internet. }\end{array}$ & 1 & 2 & 3 & 4 & 5 \\
\hline 7 & $\begin{array}{l}\text { Entiende y analiza el } \\
\text { impacto de las TIC } \\
\text { en su contexto social } \\
\text { promoviendo espacios de } \\
\text { convivencia digital. }\end{array}$ & 1 & 2 & 3 & 4 & 5 \\
\hline
\end{tabular}

Fuente: Elaboración propia

Posteriormente, se implementaron estrategias pedagógicas de uso de Tecnología y TIC específicas para el área como la creación de un blog para los estudiantes, una salida pedagógica en la que se involucran con el uso de aparatos de gimnasio y se explica el uso de los mismos a través de la grabación de videos con sus dispositivos móviles, se realiza la grabación de los grupos de trabajo en la que expresan sus opiniones con relación a las actividades desarrolladas en la clase, se emplea la App Munzee con la que deben trabajar con su teléfono 
celular y usar el GPS para lograr conseguir los códigos QR localizados, y constantemente se promovió el uso de grupos de WhatsApp para transmitir información relacionada con el área de educación física.

Luego de la implementación, se aplicó otro instrumento como postest tipo escala Likert como se observa en la tabla 2 , para medir la percepción de los estudiantes, el cual se validó por tres expertos en el área de educación física y se calculó el alfa de Cronbach para verificar su fiabilidad. Al respecto, González y Pazmiño (2015) manifiestan que el alfa de Cronbach está diseñado para evaluar el grado en que los ítems de un instrumento están correlacionados, con respecto a la utilidad estadística de Cronbach, Quero (2010) afirma que se orienta para calcular la confiabilidad de un instrumento cuyos ítems o reactivos conformen un único dominio, dominio que en nuestro caso corresponde a la formación integral e inclusión del componente Tecnológico en el área, lo que indica que este coeficiente nos permite determinar la validez de la encuesta.

Tabla 2. Matriz de Consistencia Instrumento Postest

\begin{tabular}{|c|c|c|c|}
\hline ITEM & ENUNCIADO & VARIABLE & $\begin{array}{c}\text { COMPETENCIA } \\
\text { BLANDA }\end{array}$ \\
\hline 1 & $\begin{array}{l}\text { Considera que la institución educativa le proporciona los } \\
\text { medios o herramientas necesarias para hacer uso de la } \\
\text { tecnología en las clases de educación física. }\end{array}$ & Tecnología & Empatía \\
\hline 2 & $\begin{array}{l}\text { Siente que el material de trabajo (balones, colchonetas } \\
\text { etc) de la clase de educación física se ajusta a las } \\
\text { características propias de cada disciplina para su } \\
\text { práctica favoreciendo su proceso de formación integral } \\
\text { en el área. }\end{array}$ & Tecnología & Empatía \\
\hline 3 & $\begin{array}{l}\text { Considera que con la inclusión de la Tecnología en } \\
\text { la clase de educación física se pueden desarrollar } \\
\text { patrones de movimiento, habilidades y competencias } \\
\text { motoras necesarias para la vida. }\end{array}$ & Tecnología & Autonomía \\
\hline 4 & $\begin{array}{l}\text { Hace uso de la Tecnología pensando en el desarrollo del } \\
\text { conocimiento de sí mismo, su capacidad comunicativa, } \\
\text { de relación, habilidades y destrezas motrices } \\
\text { favoreciendo su corporeidad y el trabajo en equipo. }\end{array}$ & Tecnología & $\begin{array}{l}\text { Trabajo en } \\
\text { equipo }\end{array}$ \\
\hline 5 & $\begin{array}{l}\text { Siente que la Tecnología contribuye con su proceso de } \\
\text { dar valor a la actividad física y al deporte despertando } \\
\text { interés en usted por el trabajo autónomo en el área de } \\
\text { educación física. }\end{array}$ & Tecnología & Autonomía \\
\hline 6 & $\begin{array}{l}\text { Considera que el uso de las TIC en el área de educación } \\
\text { física puede mejorar el desarrollo de la misma } \\
\text { generando ambientes caracterizados por el buen trato, } \\
\text { el respeto, el interés y la confianza afianzando sus } \\
\text { valores a partir de la actividad física. }\end{array}$ & Uso de TIC & $\begin{array}{l}\text { Trabajo en } \\
\text { equipo }\end{array}$ \\
\hline 7 & $\begin{array}{l}\text { Siente que con la inclusión de las redes sociales en el } \\
\text { área de educación física puede fomentar acciones que } \\
\text { promuevan un mejor estado de salud, actividad física y } \\
\text { una alimentación saludable. }\end{array}$ & Uso de TIC & Liderazgo \\
\hline
\end{tabular}




\begin{tabular}{|c|l|c|c|}
\hline 8 & $\begin{array}{l}\text { Acostumbra manejar medios de comunicación } \\
\text { electrónica (correo, WhatsApp, Facebook, Twitter, } \\
\text { Skype, etc.) para apropiar conceptos y principios de } \\
\text { movimiento propios del área de educación física. }\end{array}$ & Uso de TIC & Autonomía \\
\hline 9 & $\begin{array}{l}\text { Considera que el teléfono inteligente puede ser usado } \\
\text { con fines académicos en el área de educación física } \\
\text { durante la práctica de alguna disciplina deportiva o en la } \\
\text { realización de cualquier ejercicio. }\end{array}$ & Uso de TIC & Liderazgo \\
\hline 10 & $\begin{array}{l}\text { Respeta los límites legales, culturales y éticos de } \\
\text { compartir información a través de las TIC, partiendo de } \\
\text { los procesos de interacción social que se dan en el aula } \\
\text { al realizar cualquier tipo de actividad física. }\end{array}$ & Uso de TIC & Empatía \\
\hline
\end{tabular}

Fuente: Elaboración propia

Finalmente, para el análisis de los resultados se utilizó el programa SPSS para graficar, crear tablas y hacer la prueba $t$ student para comparación de medias. Para esto se promediaron las respuestas de los estudiantes a cada una de las preguntas tanto en el pretest como en el postest con la idea de presentar una variable cuantitativa continua de 1 a 5 , variable percepción, en la que 1 refleja una percepción baja respecto a la formación integral y 5 una percepción alta; se presenta un diagrama de caja y bigotes con sus respectivos datos y por último se hace una prueba de comparación de medias para poder mostrar que el tratamiento tienen efectos positivos en la percepción de la formación integral de los estudiantes.

\section{RESULTADOS}

\section{CONFIABILIDAD DE LOS INSTRUMENTOS}

Para el cálculo del alfa de Cronbach se utilizó el software SPSS versión 20, el cual arrojó un resultado de 0.946 para el pretest y de 0.795 para el postest como se muestra en la tabla 3 , y con lo cual se pudo confirmar la fiabilidad de los instrumentos presentados en las tablas 1 y 2 .

Tabla 3. Alfa de Cronbach

Alfa para el pretest

Estadisticas de fiabilidad

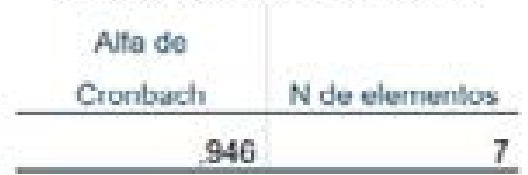

Alfa para el postest

\section{Estadisticas de fiabilidad}

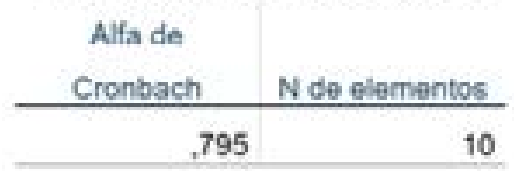

Fuente: Elaboración propia

\section{DISTRIBUCIÓN DATOS DEL PRETEST/ POSTEST}

Los datos obtenidos sobre la percepción de los sujetos frente a su grado de formación integral mediado por el componente tecnológico antes y después se pueden analizar mediante la figura 1 : 

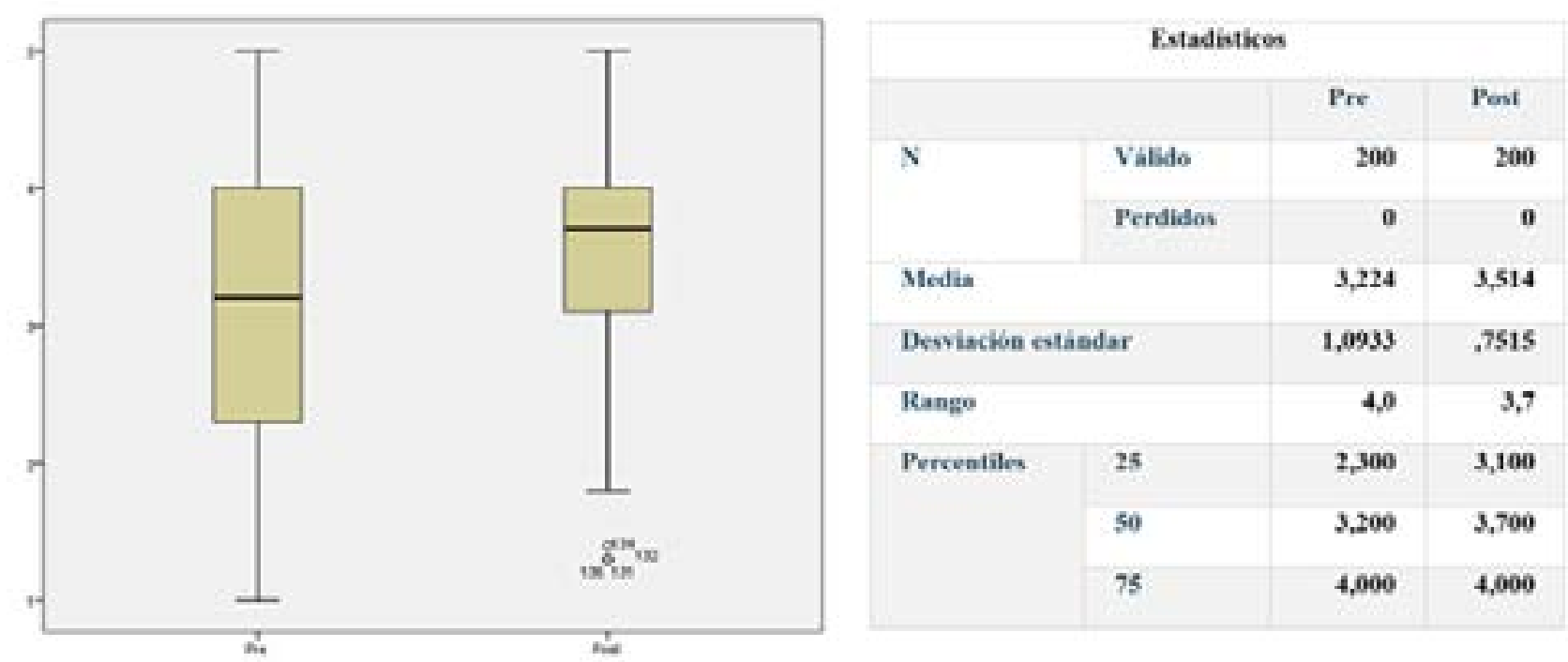

Figura 1. Percepción.

Fuente: Elaboración propia

En la figura 1 se puede apreciar una diferencia en los promedios, pasó de 3.224 a 3.514 después del tratamiento, también hay una diferencia en la distribución de la variable, así como un aumento en la mediana. Además, en la figura se observa una disminución del rango intercuartil de 1,7 en el pretest a 0,9 en el postest, este cambio indica una menor dispersión de los datos en el postest. Disminución que refleja el impacto positivo del tratamiento en el grupo, este mismo hecho puede verificarse dada la disminución en la desviación estándar de 1.09 en el pretest a 0.75 en el postest. Por otro lado, podemos notar un aumento en el primer cuartil de 2.3 a 3.1, mientras que el tercer cuartil se mantiene en 4.0, lo que provocó una asimetría en la distribución de los datos en el postest, este hecho implica entre otras cosas, una concentración del $25 \%$ de las percepciones de los sujetos sobre su grado de formación integral comprendida entre 3.7 y 4.0 , lo cual, nuevamente permite afirmar que en términos generales la intervención estratégica de inclusión del componente tecnológico fue positiva en el grupo.
Asimismo, se muestra en el postest la presencia de cuatro datos atípicos o distantes del grupo, los sujetos $131,132,134$ y 136 cuyas percepciones sobre la variable está por debajo del límite inferior en el diagrama, esto es, por debajo de 1.75 .

Ahora bien, aunque podemos notar un aumento efectivo en la media de la variable, es necesario verificar si esta diferencia es significativa, para ello se verifica la distribución normal de los datos como se observa en la prueba de Kolmogorov-Smirnov para una muestra figura 2 y posteriormente, se aplicó la prueba $\mathrm{t}$ de student para dos muestras relacionadas, estableciendo la significancia en la diferencia de las medias antes y después del tratamiento con una tolerancia del $5 \%$, en coherencia con los resultados descritos en la tabla 5 : 


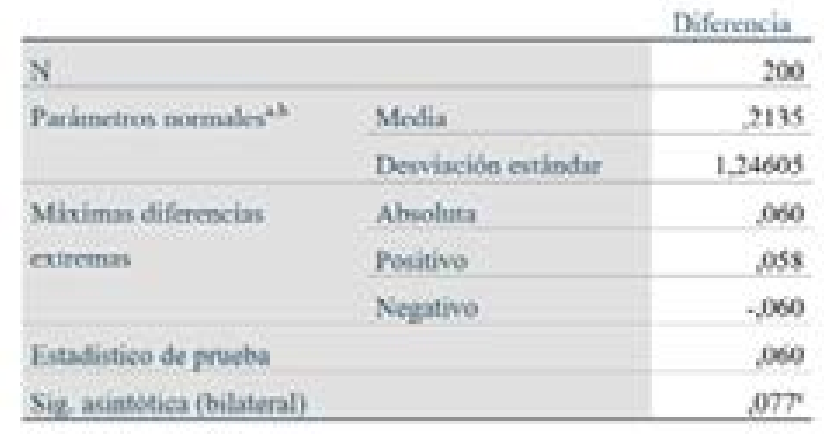

3. La distribución de procba es sormal.

h. Se calcala a partir de datos.

c. Cerreccion de significaciòa de Lilliefors.

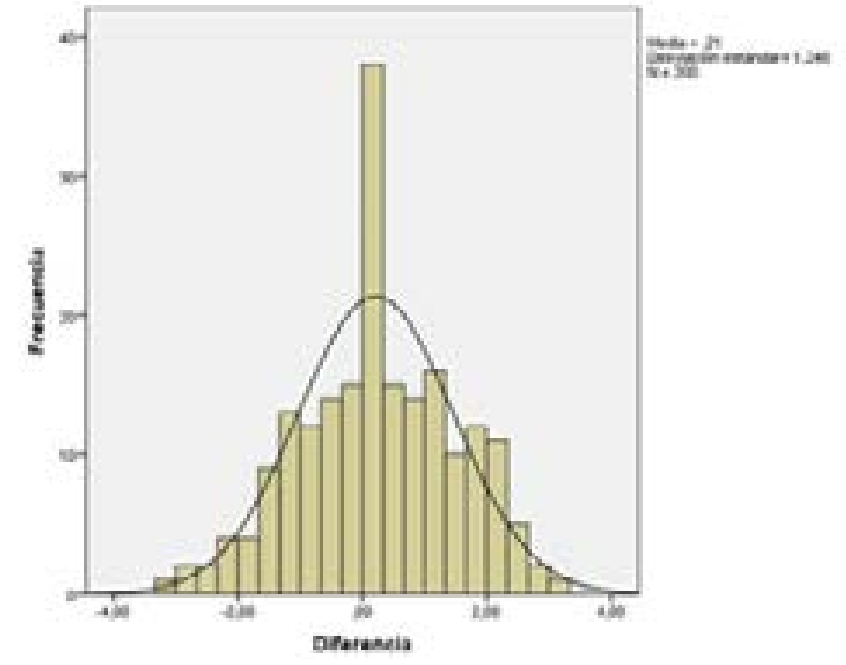

Figura 2. Prueba de Kolmogorov-Smirnov para una muestra.

Fuente: Elaboración propia

Tabla 5. Prueba t student para comparación de medias

\begin{tabular}{|c|c|c|c|c|c|c|c|c|c|}
\hline & & \multicolumn{5}{|c|}{ Diferencias emparejadas } & \multirow[t]{3}{*}{$t$} & \multirow[t]{3}{*}{ GI } & \multirow{3}{*}{$\begin{array}{l}\text { Sig. } \\
\text { (bilateral) }\end{array}$} \\
\hline & & \multirow[t]{2}{*}{ Media } & \multirow[t]{2}{*}{$\begin{array}{l}\text { Desviaciós } \\
\text { estindar }\end{array}$} & \multirow[t]{2}{*}{$\begin{array}{c}\text { Media de error } \\
\text { catindar }\end{array}$} & \multicolumn{2}{|c|}{$\begin{array}{l}95 \% \text { de intervalo de } \\
\text { confianya de la diferencis }\end{array}$} & & & \\
\hline & & & & & Inferior & Saperiar & & & \\
\hline $\begin{array}{l}\text { Par } \\
1\end{array}$ & $\begin{array}{l}\text { Pre - } \\
\text { Post }\end{array}$ & -2905 & 1,2625 & .0893 & -4665 & -1145 & $-3,254$ & 199 &, 001 \\
\hline
\end{tabular}

Fuente: Elaboración propia

Dos elementos se destacan en la tabla 5 el primero la diferencia de las medias de 0.2905 , y el segundo la significancia de 0.001 , que es menor al nivel de confianza 0.05 , entonces se puede afirmar que la diferencia de las medias antes y después de la intervención pedagógica de uso de Tecnología y TIC, es estadísticamente significativa con base en los promedios de percepción de los sujetos respecto a su formación integral.

\section{DISCUSIÓN}

Con relación a la salida pedagógica, se observó el acercamiento que tuvieron los estudiantes con la tecnología a través de reconocimiento y uso de implementos tecnológicos propios de la disciplina en el Gimnasio, pues realizaron una práctica guiada y tuvieron acceso a máquinas que normalmente no usan debido a las condiciones de la institución. El proceso de evaluación que consistió en exponer su experiencia a través de un video grabado con su teléfono celular, los estudiantes manifestaron su agrado y la motivación que generó en ellos esta salida pues no es común que se lleven a realizar este tipo de prácticas. Con esta actividad se evidenció el planteamiento de Hernández (2017) relacionado con la innovación educativa que promueve el uso de la Tecnología en el aula y con la importancia de usar elementos tecnológicos en los procesos de enseñanza y aprendizaje, ya que los estudiantes después de la práctica se mostraron más receptivos al uso de la Tecnología y adicionalmente, se comprobó la afirmación de Plaza (2013) relacionada con los cambios en los tipos de competencia y temáticas reclamadas por los estudiantes, pues ellos reflejaron un 
gran interés por dar continuidad a este tipo de actividades en el área de educación física.

El uso de la aplicación Munzee en la clase fue bastante novedoso, se observó cómo los estudiantes participaron activamente de la clase y trabajaron en equipo para conseguir los códigos QR en el menor tiempo posible. Con esta actividad se promovió la construcción de valores tal como lo plasmó Camblor (2014), ya que los estudiantes generaron un espacio de diálogo, en el cual debían seguir indicaciones y respetar la palabra del otro para poder culminar con éxito el trabajo asignado. Adicionalmente se promovieron habilidades blandas como el trabajo en equipo y el liderazgo, ya que debían trabajar en conjunto y alguien tomó la vocería para organizar el equipo en función del resultado deseado. El uso del GPS permitió la inclusión de la App en la clase, los estudiantes se desplazaron de acuerdo a la ubicación de los códigos en la institución, generando movimientos más rápidos y coordinados.

Con la inclusión de las TIC en la clase de educación física, se reforzaron los planteamientos de autores como Prat, Camerino y Coiduras (2014), quienes sostienen que el uso de herramientas tecnológicas mejoran y aportan el proceso de enseñanza y aprendizaje, se logra tener estudiantes motivados y con más receptividad a la clase educación física (Monroy, 2010; citado por Fernández y Ladrón, 2015), promueve la mejora de la actividad física con la inclusión de las TIC en el aula, pues se evidenció que a diferencia de otras actividades con el uso de las TIC se presentó una participación total de la población estudiantil en las clases; Quintero (2018) afirma, que las TIC deben ser usadas con fines didácticos en el aula donde el estudiante se compromete a través del diálogo, lo que se logró con el hecho de que los estudiantes tuvieron un paquete de datos exclusivamente para las actividades y no se presentaron dificultades pues desde el inicio se establecieron las reglas y parámetros de uso de los dispositivos móviles en las sesiones; Fernández y Ladrón (2015), establecen los móviles como una herramienta fundamental y efectivamente se vio reflejado en las sesiones pues los estudiantes participaban de manera más activa y se generan aprendizajes significativos; y Aznar, Cáceres, Trujillo y Romero (2019) los móviles efectivamente constituyen un nuevo componente motivacional, pues en las sesiones los estudiantes se vinculan a la práctica de actividad física a través de ellos.

El uso de Blog https://edufisicasanisidoro. blogspot.com/ permitió que los estudiantes incluyeran las TIC en la clase de educación física, ya que ellos son los protagonistas de la información que se carga en el mismo, adicionalmente, tienen la opción de realizar comentarios y hacer aportes, en los que discuten con miembros de su comunidad educativa sobre las temáticas desarrolladas en el curso.

Con relación a la pregunta 1 que hizo referencia a si la institución les proporciona los medios o las clases de educación física, se encontró que el $45 \%$ de los encuestados considera que nunca y casi nunca cuentan con las herramientas necesarias para su uso en las clases. La pregunta 2 cuestionó sobre las características del material de trabajo en la clase de educación física, el $52 \%$ de los estudiantes siente que casi siempre y siempre cuentan con el material adecuado para cada disciplina deportiva en las clases de educación física. De acuerdo con los resultados obtenidos de estas preguntas, la institución debe mejorar según Fernández y Ladrón (2015), ya que esta debe evolucionar y brindar los medios y herramientas necesarias a los estudiantes para mejorar sus procesos de enseñanza y aprendizaje. Con relación a las características del material de trabajo, los estudiantes sienten que son utilizados en función de sus procesos de enseñanza ya que se sienten cómodos tanto con las colchonetas, 
balones y demás material utilizado en las prácticas, de acuerdo a Hernández (2017). Estas preguntas apuntan a establecer el nivel de empatía de los estudiantes con relación al uso de la Tecnología en las clases de educación física, en este sentido Camblor (2014) afirma que la Tecnología acerca empáticamente a los sujetos en formación.

La pregunta 3 hizo referencia a la inclusión de la Tecnología con el fin de desarrollar patrones de movimiento, habilidades y competencias propias del área, donde el $59.5 \%$ de los encuestados considera que, a través del uso de la Tecnología en las clases de educación física, casi siempre y siempre desarrollan estos patrones, adicionalmente, desarrollan autonomía en las clases y se prepara los estudiantes en competencias blandas como lo plantea Gómez (2018).

La pregunta 4 objetó sobre el uso que se da a la tecnología pensando en el desarrollo de habilidades y trabajo en equipo, el $71 \%$ de los estudiantes manifestaron que casi siempre y siempre desarrollan habilidades y trabajan en equipo en la clase cuando se involucra la Tecnología. El Trabajo en equipo que se desarrolla de acuerdo con Tapia (2018), es necesario para establecer buenas relaciones sociales en el aula.

La pregunta 5 hizo referencia a la contribución de la Tecnología para dar valor a la actividad física y al trabajo autónomo en el área, el 63.5\% de los estudiantes siente que casi siempre y siempre el uso de la Tecnología contribuye con este proceso. De acuerdo al resultado se promueve el desarrollo de habilidades blandas como lo plantea García (2018), que para este caso hace referencia al trabajo autónomo de los estudiantes en las clases.

La pregunta 6 hizo énfasis en el uso de las TIC como herramienta que favorece la formación en valores desde el área de educación física, el $64.5 \%$ de los estudiantes considera que casi siempre y siempre el uso de las TIC mejora la clase y genera espacios de respeto entre los compañeros, favoreciendo el desarrollo de habilidades socioemocionales y de identificación de las de otros, como lo establece el (BID, 2017; citado por Gómez, 2019).

La pregunta 7 cuestionó acerca de la inclusión de las redes sociales como herramienta de promoción de acciones que mejoren la salud, la actividad física y una alimentación saludable, el $52 \%$ de los estudiantes siente que casi siempre y siempre las redes sociales contribuyen con la promoción de las actividades mencionadas y promueven el desarrollo de liderazgo, haciendo énfasis en lo planteado por Equihua (2007), ya que se está logrando un desarrollo equilibrado y armónico del estudiante a partir del uso de las TIC en el área, generando un impacto directo en el proceso de formación integral.

La pregunta 8 se refirió al manejo de medios de comunicación electrónicos para apropiar conceptos propios del área, el 39\% de los estudiantes casi siempre o siempre usa correo, WhatsApp, Facebook, Twitter, Skype, preferentemente para tal fin, mientras que el $32.5 \%$ casi nunca o nunca lo hace. La pregunta 9 cuestionó el uso del teléfono móvil con fines académicos en el área de educación física, el $52.5 \%$ de los estudiantes considera que casi siempre o siempre el teléfono celular puede ser usado en clase para realizar prácticas deportivas o con fines académicos y tan solo el $25 \%$ considera que casi nunca o nunca puede ser usado para tal fin. En este sentido, Aznar, Cáceres, Trujillo y Romero (2019) sostienen que los dispositivos móviles son un potente recurso para la mejora y aumento de la práctica deportiva, lo cual se ve reflejado en el resultado de la pregunta.

La pregunta 10 deliberó sobre el respeto por las normas, límites legales, culturales y éticos de la información que se comparte a través de 
las TIC a partir de los procesos de interacción social que se dan en las clases de educación física, el $76 \%$ de los estudiantes respetan casi siempre o siempre las normas al difundir información a través de las TIC, el 19\% lo realiza ocasionalmente, y tan solo el $5 \%$ lo hace casi nunca o nunca, el resultado refleja el enriquecimiento de los procesos de socialización propuestos por Orozco (2008).

\section{CONCLUSIONES}

A partir del análisis de los resultados y con la aplicación de estrategias de inclusión del componente Tecnológico en las clases de educación física como la práctica de Gimnasio, la grabación de videos tanto de experiencias como de explicación de ejercicios, el uso de una App como Munzee, el dispositivo móvil, el GPS, la inclusión de las redes sociales como herramienta de comunicación de carácter académico, el uso de materiales como colchonetas, balones e implementos con la tecnología adecuada para la disciplina deportiva a practicar y la creación del Blog, permiten que los estudiantes se formen integralmente, desarrollen competencias blandas como la empatía, la autonomía, el liderazgo y el trabajo en equipo que van a contribuir con el fortalecimiento de sus procesos de socialización impactando además su proceso de enseñanza y aprendizaje.

La inclusión de las TIC en el aula resulta bastante complicada, en razón a que son empleadas de una manera más recreativa que académica, pero con la orientación adecuada, las pautas de uso y normatividad clara, se convierten en una gran herramienta de trabajo en las clases de educación física, sobre todo para motivar a los estudiantes que regularmente no se interesan por las temáticas de la clase, pues se presentan actividades novedosas y diferentes para ellos, promoviendo un aumento significativo en la práctica de actividad física.
Por otra parte, es preciso que la institución educativa se vincule más al uso del componente Tecnológico ya que no es suficiente dotar a los estudiantes de material óptimo para la práctica de sus clases, sino que se hace necesario que brinde la posibilidad de acceso a redes de internet que permitan un acercamiento a las distintas App que se pueden incluir en las prácticas de educación física. Con ello, se pueden mejorar aspectos relacionados con el manejo de medios de comunicación electrónicos en la clase, para hacer apropiación de conceptos propios de esta y que se siga favoreciendo los espacios de formación en valores, el uso el móvil y redes sociales con fines académicos.

Conocer las percepciones de los estudiantes constituye un factor muy relevante para evaluar el proceso de formación y las estrategias implementadas en el aula, pues es a través de ellas que se pueden mejorar e indagar sobre las necesidades propias de su edad en relación al contexto, ya que un estudiante motivado y con una experiencia significativa positiva está más receptivo a realizar nuevas actividades y lógicamente, se tendrán clases más didácticas y llamativas para ellos, pues la Tecnología debe ser vista, trabajada y promovida como una herramienta educativa para el área de educación física.

Finalmente, se concluye que las estrategias empleadas si tiene efectos positivos en tanto que las medias de las percepciones de los sujetos sobre su formación integral aumentaron significativamente.

\section{REFERENCIAS BIBLIOGRÁFICAS}

Aznar, I., Cáceres, M., Trujillo, J., y Romero J. (2019). Impacto de las apps móviles en la actividad física un meta-análisis. Revista Retos: nuevas tendencias en educación física, deporte y recreación. ISSN 1579-1726, №. 36, págs. 52-57. https://dialnet.unirioja.es/servlet/ articulo?codigo $=6770637$ 
Barquero, A., y Calderón, F., (2016). Influencia de las nuevas Tecnologías en el desarrollo adolescente y posibles desajustes. Revista Cúpula n 30 (2): 11-25. https://www.binasss. sa.cr/bibliotecas/bhp/cupula/v30n2/art02.pdf

Bustos, P. (2014). Percepciones de los alumnos en relación a sus aprendizajes mediante la utilización de las Tecnologías de la Información y Comunicación (TIC) en el área de Historia y Ciencias Sociales. Congreso Iberoamericano de Ciencia, Tecnología, Innovación y Educación. Buenos Aires: Argentina, ISBN: 978-84-7666-210-6 - Artículo 488. https:// www.oei.es/historico/congreso2014/contenedor. php?ref=memorias

Camblor, E. (2014). Prácticas de la educación física. La Plata: Universidad Nacional de La Plata. Facultad de Humanidades y Ciencias de la Educación. (Colectiva y Monográfica; 2). En Memoria Académica. http://www.memoria. fahce.unlp.edu.ar/libros/pm.393/pm.393.pdf

Campo, R. (1999). Formación Integral: modalidad de educación posibilitadora de lo humano. Bogotá D.C., Tercera reimpresión. Editorial Pontificia Universidad Javeriana.

Equihua, A. (2007). La Educación Física en la Formación Integral de los alumnos de primaria. Zamora: Universidad Pedagógica Nacional. http://200.23.113.51/pdf/25310.pdf

Fernández, R., Herrera, J., y Navarro, R. (2015). Las TIC en Educación Física desde la perspectiva del alumnado de Educación Primaria. Sportis Scientific Technical Journal, 1 (2), 141-155. https://ruc.udc.es/dspace/bitstream/ handle/2183/17666/SPORTIS_1_2_2015_5. pdf? sequence $=1 \&$ is Allowed $=y$

Fernández, C., y Ladrón, L. (2015). El uso de las TIC en la Educación Física actual. E-motion. Revista de Educación, Motricidad e Investigación $\mathrm{n}^{\circ}$ 5, pp. 17-30. ISSN: 2341-1473. https://www. researchgate.net/publication/322132086 El uso de las TIC en la Educacion Fisica actual

Gamboa, M., Briceño, J., y Camacho, J. (2015). Caracterización de estilos de aprendizaje y canales de percepción de estudiantes universitarios. Opción, vol. 31, núm. 3, 2015, pp. 509-527 Universidad del Zulia Maracaibo, Venezuela. http://www.redalyc.org/articulo. oa?id=31045567026

García, B. (2018). Las habilidades socioemocionales, no cognitivas o "blandas": aproximaciones a su evaluación. Revista Digital Universitaria (RDU). Vol. 19, núm. 6 noviembre - diciembre. http://doi.org/10.22201/ codeic.16076079e.2018.v19n6.a5

Gómez, M. (2019). Las habilidades blandas competencias para el nuevo milenio. Revista Divulgare. Publicación semestral No. 11. Universidad Autónoma del Estado de Hidalgo. México. https://repository.uaeh.edu.mx/revistas/ index.php/divulgare/article/view/3760/5951

González Alonso, J., \& Pazmiño Santacruz, M. (2015). Cálculo e interpretación del Alfa de Cronbach para el caso de validación de la consistencia interna de un cuestionario, con dos posibles escalas tipo Likert. Revista Publicando, 2(1), 62-67. https://www.ssoar. info/ssoar/bitstream/handle/document/42382/ ssoar-revpublicando-2015-1-gonzalez_alonso jorge_et_al-Calculo_e_interpretacion_del.pd f? sequence $=1 \&$ is Allowed $=y \&$ Inkname $=$ ssoar revpublicando-2015-1-gonzalez_alonso_jorge et al-Calculo e interpretacion del.pdf

Hernández, R., (2017). Impacto de las TIC en la educación: Retos y Perspectivas. Propósitos y Representaciones, v. 5, n. 1, p. 325-347, abr. 2017. ISSN 2310-4635. http://revistas.usil.edu. pe/index.php/pyr/article/view/149

Mateos, T. (2009). La percepción del contexto escolar. Una imagen construida a partir de las experiencias de los alumnos. Revista 
Cuestiones Pedagógicas, 19, 2008/2009, p 285300. Secretariado de publicaciones Universidad de Sevilla. http://institucional.us.es/revistas/ cuestiones/19/16Mateos.pdf

Orozco, L. (2008). La formación integral. Mito y realidad. Revista Universitas No. 10. Universidad Politécnica Salesiana de Ecuador. https:// revistas.ups.edu.ec/index.php/universitas/ article/view/10.2008.07

Ortega, C., Febles, J. y Estrada, V. (2016). Fundamentación teórico-metodológica de una estrategia para desarrollar habilidades blandas desde la enseñanza inicial. Revista Ecociencia. . http://ecociencia.ecotec.edu.ec/articulo?ida=69

Prat, A., Camerino, O., y Coiduras, J. (2013). Introducción de las TIC en educación física. Estudio descriptivo sobre la situación actual. Revista Apuntes Educación Física y Deportes n. - 113, 3.er trimestre (julio-septiembre), pp. 3744 ISSN-1577-4015. http://www.observesport. com/desktop/images/docu/3walelni.pdf

Quero, M. (2010). Confiabilidad y coeficiente Alpha de Cronbach. Revista Telos, 12 (2), 248252. Universidad Rafael Belloso. Recuperado de http://www.redalyc.org/pdf/993/99315569010. $\underline{p d f}$

Quintero, L. (2018). Claves para la integración y el uso didáctico de los dispositivos móviles en las clases de educación física. Revista Acción Motriz ISSN-e 1989-2837, №. 20, págs. 17-26. http://www.Dialnet-ClavesParaLalntegracionYEl UsoDidacticoDeLosDisposi-6435705.pdf

Plaza, L. (2013). Uso de las TIC en el área de educación física. Universidad Internacional de la Rioja. https://reunir.unir.net/bitstream/ handle/123456789/2057/2013_09_19 TFM_ESTUDIO_DEL_TRABAJO. pdf? sequence $=1$ \&isAllowed $=y$
Rivera, C. (1995). ¿Cómo se da la percepción de la escuela en los adolescentes? una propuesta teórica. Revista Educación. Vol. IV. No 8. Septiembre. http://revistas.pucp.edu.pe/index. php/educacion/article/view/5094

Rojano, D., (2010). Uso de recursos TIC en la clase de Educación Física. Una experiencia positiva con el vídeo digital y el salto vertical. Revista Retos, Nuevas Tendencias en Educación Física, Deporte y Recreación, núm. 17, enerojunio, 2010, pp. 107-110. https://recyt.fecyt.es/ index.php/retos/article/view/34687/18794

Singer, M., Guzmán, R. y Donoso, P. (2009). Entrenando Competencias Blandas en Jóvenes. Escuela de Administración Pontificia Universidad Católica de Chile. INACAP. http://www.inacap. cl/tportal/portales/tp90b5f9d07o144/uploadlmg/ File/PDF/Entre nando Competencias Blandas en Jovenes.pdf

Soto A., (2000). Educación en tecnología: Un reto y una exigencia social. Ed Magisterio pág., 26. Bogotá - Colombia.

Tapia, L. (2018). Las habilidades blandas y la convivencia escolar de los estudiantes de la institución educativa 2051 El ProgresoCarabayllo 2018. Repositorio Universidad César Vallejo. Perú. http://repositorio.ucv.edu. pe/bitstream/handle/UCV/23844/Tapia_TLA. pdf? sequence $=1$ \&isAllowed $=y$ 